\title{
QUALIDADE DE FRUTOS DE PITAYA EM FUNÇÃO DA ÉPOCA DE POLINIZAÇÃO, DA FONTE DE PÓLEN E DA COLORAÇÃO DA COBERTURA ${ }^{1}$
}

\author{
ADRIANA DE CASTRO CORREIA DA SILVA², ANTONIO BALDO GERALDO MARTINS ${ }^{3}$, \\ LUDMILLA DE LIMA CAVALLARI ${ }^{4}$
}

RESUMO-A pitaya (Hylocereus undatus) é uma cactácea propagada vegetativamente, o que resulta em pequena ou nula variabilidade nos plantios comerciais. Devido a diversos estudos mostrarem que a espécie é auto ou parcialmente incompatível, realizou-se este trabalho com o intuito de verificar a eficiência de polinização da pitaya autofecundada e utilizando-se de pólen de H. polyrhizus e Selenicereus setaceus, além da influência da época do ano e da coloração da cobertura da tela plástica na qualidade dos frutos. O experimento foi realizado na Área de Fruticultura da Faculdade de Ciências Agrárias e Veterinárias - UNESP, Campus de Jaboticabal, num delineamento experimental inteiramente casualizado, em esquema fatorial 3 X 2 X 2 (três espécies doadoras de pólen X duas colorações de cobertura de sombrite, branca e preta, com $50 \%$ de sombreamento $X$ duas épocas do ano), com quatro repetições para cada espécie doadora de pólen, em cada uma das coberturas. As flores foram emasculadas em duas épocas (março e abril) e polinizadas manualmente. As variáveis avaliadas foram porcentagem de pegamento dos frutos, características físicas e químicas dos frutos (comprimento e diâmetro, massas total, de polpa e de casca, \% de polpa, espessura da casca, $\mathrm{pH}$, teor de sólidos solúveis ( $\left.{ }^{\circ} \mathrm{Brix}\right)$, teor de vitamina $\mathrm{C}$, acidez titulável, índice de maturação - ST/AT) e dias da antese até a colheita. Não houve frutificação nas flores autopolinizadas, enquanto as polinizadas com pólen de $H$. polyrhizus e $S$. setaceus obtiveram $100 \%$ de pegamento. Pode-se concluir que há necessidade de interplantio de plantas de $H$. undatus (clone avaliado) com H. polyrhizus ou S. setaceus para a ocorrência de frutificação, sendo que a utilização de $H$. polyrhizus como doadora de pólen proporciona a obtenção de frutos com maior massa e menor acidez. As condições climáticas ocorridas durante o desenvolvimento dos frutos, nas épocas estudadas, afetaram a qualidade dos frutos.

Termos para indexação: frutificação, sombreamento, polinização manual, Hylocereus undatus.

\section{FRUIT QUALITY OF PITAYA AS A FUNCTION OF POLLINATION TIME, POLLEN SOURCE AND ENVIRONMENT FOR CULTIVATION}

\begin{abstract}
The pitaya (Hylocereus undatus) is a cactaceae vegetative propagated, that results in little or no variability in commercial plantations. Due to several studies shown that the specie is auto or relatively self-incompatible, this study was conducted with the objective to check the efficiency of self pollination in red pitaya and utilizing pollen from H. polyrhizus and Selenicereus setaceus, and the effect of time and the plastic screen cover color, on fruit quality. The research was realized at the Faculdade de Ciências Agrárias e Veterinárias - UNESP, Jaboticabal Campus, Brazil, in a completely randomized design, factorial 3 X 2 X 2 (three pollen sources $\mathrm{X}$ two environment for cultivation - under plastic screen, black and white, with $50 \%$ of shade $\mathrm{X}$ two periods) and four replicates for each pollen source, in each of the coverage. The flowers were emasculated in two periods (March and April) and hand pollinated. The variables evaluated were percentage of fruit set and physical and chemical characteristics (length, diameter, total weight, flesh weight, skin weight, percentage of flesh pulp, peel thickness, $\mathrm{pH}$ value, total soluble solids content ( $\left.{ }^{\circ} \mathrm{Brix}\right)$, ascorbic acid content, titratable acidity, ST/AT ratio) and the period from pollination to fruit ripening (days). There was no fruit set in auto-pollinated flowers, although cross-pollinated flowers with $H$. polyrhizus and $S$. setaceus pollen the fruit set was $100 \%$. It can be concluded that is necessary mixing plants of H. undatus with different species (H. polyrhizus or S. setaceus) in order to occur fruit set, and the use of $H$. polyrhizus pollen as donor provides heavier fruits and with less acidity. The environmental conditions occurring during fruit development at the evaluated period, influenced fruit quality.
\end{abstract}

Index terms: fruit set, shading, hand pollination, Hylocereus undatus.

\footnotetext{
'(Trabalho 054-11). Recebido em: 12-01-2011. Aceito para publicação 08-07-2011.

${ }^{2}$ Eng. Agr., aluna de pós-graduação em Agronomia -Produção Vegetal - FCAV/UNESP - Dept ${ }^{\circ}$ de Produção Vegetal. Via de acesso Prof. Paulo Donato Castellane, s/n. CEP 14884-900 Jaboticabal-SP. e-mail: dri_ubatuba@yahoo.com.br, FAPESP: processo 2008/56615-5

${ }^{3}$ Eng. Agr., Prof. Dr., Dept ${ }^{\circ}$ de Produção Vegetal. FCAV/UNESP, e-mail: baldo@fcav.unesp.br

${ }^{4}$ Eng. Agr., ,Msc., Dept ${ }^{\circ}$ Produção Vegetal - FCAV/UNESP. e-mail: milla.cavallari@hotmail.com. Bolsista FAPESP
} 


\section{INTRODUÇ̃̃O}

O consumo de frutas exóticas tem apresentado aumento nos últimos anos, despertando interesse tanto do consumidor local quanto do estrangeiro. Dentre as frutíferas exóticas com grande potencial de comercialização, encontra-se a pitaya [Hylocereus undatus (Haworth) Britton \& Rose], cactácea nativa das florestas tropicais das Américas. Segundo dados da CEAGESP, no ano de 2009, foram comercializadas 115 toneladas de frutos, enquanto em 2010, até novembro, a quantidade comercializada já passava de 138 toneladas (SILVA, 2011).

A pitaya é uma planta perene, que cresce comumente sobre árvores ou pedras, com caule classificado morfologicamente como cladódio, com forma triangular e espinhos de 2 a $4 \mathrm{~mm}$ de comprimento (CANTO et al., 1993). Os cladódios são suculentos, de coloração verde, com pouco mais de $20 \mathrm{~cm}$, em média, de comprimento e de 5 a $7 \mathrm{~cm}$ no segmento transversal (DONADIO, 2009).

Dos cladódios são originadas numerosas raízes adventícias que contribuem na absorção de nutrientes e na fixação da planta ao suporte onde é conduzida, mas que não tem ação parasítica (ORTIZ, 2000). O sistema radicular é superficial, fasciculado, com grande capacidade de extração de nutrientes do solo mesmo quando estes se encontram em baixas concentrações (LE BELLEC et al., 2006).

As flores são hermafroditas, grandes (cerca de $30 \mathrm{~cm}$ de comprimento), aromáticas e brancas (BARBEAU, 1990). Em H. undatus, os botões florais são formados pouco antes da antese, apresentando um rápido desenvolvimento, cerca de três semanas (NERD et al., 2002a). O florescimento ocorre durante a noite, e as flores se abrem uma única vez.

A propagação da pitaya pode ser feita por meio de sementes ou de maneira vegetativa, sendo comumente realizada através da estaquia. O método sexual é utilizado quando se objetiva obtenção de variabilidade, sendo utilizado principalmente em programas de melhoramento para a seleção de materiais com características desejáveis, como produtividade, aparência externa, coloração de polpa e melhor adaptação às diferentes condições climáticas (ANDRADE et al., 2008). Segundo Marques et al. (2008), as sementes podem ser armazenadas em câmara fria por até um ano, sem prejuízos à porcentagem de germinação e apresentam alta viabilidade. Porém, para que cheguem à idade produtiva, as plantas oriundas de sementes demoram de três a sete anos (ORTIZ, 2000).

As plantas originadas por estaquia iniciam o florescimento após um ou dois anos depois do plan- tio. Além da precocidade na produção, a propagação por estaquia, de cladódios inteiros ou segmentados, é a forma mais prática para se obterem plantações uniformes, fator importante para cultivos comerciais, devido à manutenção das características fenológicas e de qualidade de frutos, necessárias ao mercado (GUNASENA et al., 2007; ANDRADE et al., 2007).

Por ser oriunda de florestas úmidas da América tropical e subtropical, a pitaya pode sofrer grandes danos e até morrer quando cultivada sob luminosidade intensa. Em várias destas cactáceas, a atividade fotossintética e o crescimento das plantas inibem-se quando crescem em locais expostos à radiação solar total (RAVEH et al., 1998). Para seu melhor desenvolvimento, Mizrahi e Nerd (1999) recomendam que seu cultivo seja feito sob sombreamento entre 30 a $60 \%$, dependendo da espécie e do local. Em estudo realizado em Israel, estes autores observaram que injúrias causadas pelo frio são frequentes quando a temperatura é inferior a $4^{\circ} \mathrm{C}$, embora quando a temperatura se eleva, a recuperação é rápida. Algumas espécies, como $H$. polyrhizus, são mais tolerantes à luz, pois apresentam uma cobertura de cera, que evita a exposição direta dos estômatos à radiação solar (MIZRAHI; NERD, 1999).

Estudos de sistemas reprodutivos conduzidos em Israel mostram que Hylocereus spp. é auto ou parcialmente autoincompatível (NERD et al., 2002b; LE BELLEC, 2004) e pólen externo (de outra espécie de Hylocereus) é necessário para que ocorra frutificação comercial, além de as condições climáticas também poderem afetar diretamente a compatibilidade (NERD et al. 2002b). A polinização artificial pode apresentar diferentes resultados, dependendo da cultivar doadora de pólen. Neste sentido, a polinização manual com diferentes genótipos (e até espécies) ajudaria no incremento da frutificação e no tamanho do fruto (massa) (LE BELLEC, 2004; LICHTENZVEIG et al., 2000). Assim, uma produção de frutos com valor comercial pode ser conseguida com a técnica de polinização cruzada a partir de clones compatíveis, com o plantio de diversos genótipos e polinização manual. A origem do pólen pode também influenciar no tempo entre a polinização e a colheita do fruto. Este fenômeno foi previamente observado em H. polyrhizus (MIZAHARI et al., 2004).

Este experimento teve o objetivo de avaliar a polinização da pitaya (Hylocereus undatus) com o próprio pólen e com pólen de H. polyrhizus e Selenicereus setaceus, a influência da época de polinização, da coloração da cobertura de tela plástica sobre o vingamento e a qualidade dos frutos. 


\section{MATERIAL E MÉTODOS}

O experimento foi conduzido durante os meses de março a maio de 2010, na área experimental do Departamento de Produção Vegetal da Faculdade de Ciências Agrárias e Veterinárias - UNESP - Câmpus de Jaboticabal-SP, com clone de pitaya (H. undatus) oriundo de plantio comercial da região de Itajobi SP, com 7 anos de idade. As plantas são cultivadas tutoradas, sob cobertura de tela plástica, com $50 \%$ de sombreamento, que cobre apenas a parte superior da planta, evitando exposição direta das plantas ao sol no período mais quente do dia, em espaçamento 2,5 $\mathrm{X}$ 4,0 m. Escolheu-se a coloração branca de tela, por esta refletir a luminosidade, e a preta, por ter efeito contrário. O clima da região, segundo a classificação de Köeppen, é Cwa (subtropical), apresentando uma temperatura média anual de $22^{\circ} \mathrm{C}$.

$\mathrm{O}$ delineamento experimental utilizado foi o inteiramente casualizado, em esquema fatorial 3X2X2 (três espécies doadoras de pólen $-H$. undatus, H. polyrhizus e $S$. setaceus X duas colorações de cobertura - branca e preta $\mathrm{X}$ duas épocas de polinização - março e abril), com 4 repetições para cada espécie utilizada como polinizadora, sendo uma flor por repetição, em cada uma das coberturas. Procurouse polinizar as flores que estivessem mais próximas umas das outras para se evitar o efeito do ambiente.

Polens de $H$. undatus, $H$. polyrhizus e Selenicereus setaceus foram coletados de coleção mantida no Ripado de Fruticultura da FCAV/UNESP, e armazenados em frascos plásticos. As polinizações ocorreram no mesmo dia da coleta dos polens, nos dois ambientes avaliados, e nas duas épocas.

No dia da antese, que ocorre no final da tarde, as flores de pitaya (H. undatus) foram emasculadas $\mathrm{e}$ polinizadas manualmente com o pólen das espécies doadoras, colocando-os em contato com o estigma da flor receptora e, após a polinização, foram cobertas com saquinho do tipo TNT (tecido não tecido), para evitar contaminação com pólen externo e lavagem por chuva. Os saquinhos foram retirados após uma semana da realização da polinização. $\mathrm{O}$ ponto de colheita foi determinado visualmente, de acordo com a coloração da casca, quando esta se tornou totalmente rosa.

Foram avaliadas a porcentagem de pegamento dos frutos, dias da antese até a colheita e características físicas e químicas - comprimento e diâmetro (medidos utilizando-se de paquímetro digital, e expresso em centímetros), massas total, de casca e de polpa (mensurados utilizando-se de balança digital, e expresso em gramas), rendimento de polpa (obtido através da relação $\left\{[(\mathrm{MT}-\mathrm{MC} /) \mathrm{MT}]^{*} 100\right\}$, em que
MT é massa total e MC é massa de casca, e expressa em porcentagem,), espessura de casca (mensurada com um paquímetro digital, em dois pontos da casca, e expressa em milímetros), $\mathrm{pH}$, vitamina $\mathrm{C}$ ( $\mathrm{mg}$ de ácido ascórbico/100mL de suco), acidez titulável (g de ácido málico/100mL de suco), sólidos solúveis ( ${ }^{\circ}$ brix), e índice de maturação (obtido através da relação entre acidez titulável/teor de sólidos solúveis) dos frutos colhidos. As avaliações químicas quanto ao $\mathrm{pH}$, acidez titulável e teor de vitamina $\mathrm{C}$ foram realizadas seguindo-se as metodologias preconizadas pelo Instituto Adolfo Lutz (1985). O teor de sólidos solúveis foi obtido utilizando-se de refratômetro digital.

Os resultados foram submetidos à análise de variância, e as médias, comparadas pelo teste de Tukey, a 5\% de probabilidade. Para fins de análise estatística, os dados de porcentagem de polpa foram transformados em arc- sen $\sqrt{x / 100}$.

\section{RESULTADOS E DISCUSSÃO}

Não houve frutificação nas flores autopolinizadas, indicando auto-incompatibilidade, embora Pushpakumara et al. (2005), em estudo conduzido em Sri Lanka, tenham obtido $100 \%$ de frutificação em flores autopolinizadas, resultado também obtido por Lone et al. (2010). Em contrapartida, Lichtenzveig et al. (2000) afirmam que, em Israel, a polinização manual cruzada de $H$. undatus e $H$. polyrhizus é essencial para incrementar a frutificação, pois são autoincompatíveis. Flores polinizadas com pólen de Hylocereus polyrhizus e Selenicereus setaceus obtiveram $100 \%$ de pegamento, valor também encontrado por Pushpakumara et al. (2005). Weiss et al. (1994) reportam que $H$. undatus não é capaz de produzir frutos através de autopolinização natural, além de produzir um reduzido número de sementes através da autopolinização manual $(50-79,6 \%$ de frutificação), e que quando a polinização é cruzada, obti+veram-se $100 \%$ de frutificação. Provavelmente, as divergências encontradas pelos trabalhos citados devem-se à utilização de clones diferentes.

Houve influência significativa quanto à origem do pólen utilizado para massa total, massa de casca, de polpa e acidez titulável. Para o fator cobertura, houve significância quanto ao teor de vitamina $\mathrm{C}$ e dias até a colheita. Para época, houve diferenças quanto à massa de polpa, espessura de casca, \% de polpa, vitamina $\mathrm{C}$ e dias até a colheita (Tabelas 1 e 2).

Observa-se que o cruzamento com $H$. polyrhizus resultou em frutos com maior massa total, de casca e polpa, para as duas épocas e coberturas 
avaliadas. O peso médio de frutos obtidos com pólen de $S$. setaceus foi de 561,25 gramas, enquanto os frutos resultantes de polinização com $H$. polyrhizus foi de 716,56 gramas, um incremento de massa superior a $25 \%$. Apesar de a massa de casca ser superior em frutos obtidos a partir de pólen de $H$. polyrhizus, não houve reflexo na diminuição da porcentagem de polpa, pois também houve um incremento concomitante na massa de polpa.

A polinização das flores em abril resultou em frutos com casca mais grossa, porém uma massa de polpa menor que os polinizados em março, resultando em menor porcentagem de polpa nos frutos colhidos.

A utilização de pólen proveniente de flores de S. setaceus resultou em frutos mais ácidos, quando comparados com os polinizados com $H$. polyrhizus (Tabela 2), porém dentre os valores comumente encontrados para a cultura $-0,33$ a $0,40 \mathrm{~g}$ de ácido málico/100mL de polpa (SINTZING et al., 2003, citado por LE BELLEC et al., 2006; CENTURIÓN et al., 2008).

$\mathrm{O}$ teor de vitamina $\mathrm{C}$ foi maior nos tratamentos com cobertura clara, e em abril. Embora a luz não seja essencial para a síntese de ácido ascórbico nos vegetais, a sua intensidade e a duração de exposição durante a estação de crescimento têm influência definida na quantidade de ácido ascórbico formado (CHITARRA; CHITARRA, 2005). No entanto, para Lee e Kader (2000), a luminosidade, durante este período, influencia na biossíntese do ácido ascórbico, que é sintetizado a partir dos açúcares produzidos na fotossíntese. Assim, o menor teor de ácido ascórbico obtido nos frutos sob cobertura escura, provavelmente, é causa da menor luminosidade ocorrida neste ambiente. Frutos crescidos em diferentes épocas podem ter características químicas e bioquímicas diferentes, em função do fotoperíodo, intensidade luminosa e precipitação. Nomura et al. (2005), estudando mudanças sazonais na coloração da casca, açúcares e ácidos em H. undatus, observaram diferenças entre frutos polinizados no verão e no outono. Frutos colhidos no outono obtiveram maior teor de sólidos solúveis que os colhidos no verão.

O tipo de cobertura e a época também influenciaram no número de dias até a colheita, ocorrendo um ciclo maior nos frutos polinizados no mês de abril (43 dias -7 dias a mais que os frutos polinizados no mês de março). A cobertura escura também resultou num aumento do ciclo, em dois dias, em relação aos frutos cultivados sob cobertura clara (Tabela 2). Centurión et al. (2008) encontraram que, para uma temperatura média de $26,1^{\circ} \mathrm{C}$ e $73,9 \mathrm{~mm}$ de precipitação, a maturação de frutos de pitaya ocorreu entre 25 e 31 dias após a abertura floral. Segundo Nerd et al. (2002b), o tempo da antese à maturação depende da temperatura. Foram registradas menores temperaturas durante o desenvolvimento dos frutos na segunda época de polinização (Figura 1).

As condições climáticas ocorridas durante o desenvolvimento dos frutos, nas épocas estudadas, afetaram a qualidade dos frutos.

TABELA 1 - Comprimento, diâmetro, massa total, massa de casca, massa de polpa, espessura de casca e \% de polpa de pitaya, resultantes dos cruzamentos realizados, em dois tipos de cobertura e duas épocas. Jaboticabal-SP, 2010.

\begin{tabular}{|c|c|c|c|c|c|c|c|}
\hline Fator de variação & $\begin{array}{r}\text { Comp } \\
(\mathrm{mm})\end{array}$ & $\begin{array}{l}\text { Diam } \\
(\mathrm{mm})\end{array}$ & $\begin{array}{c}\text { Massa } \\
\text { total }(\mathrm{g})\end{array}$ & $\begin{array}{c}\text { Massa } \\
\text { casca }(\mathrm{g})\end{array}$ & $\begin{array}{c}\text { Massa } \\
\text { polpa (g) }\end{array}$ & $\begin{array}{l}\text { Esp. } \\
\text { casca } \\
(\mathrm{mm})\end{array}$ & $\%$ polpa \\
\hline $\begin{array}{l}\text { Origem do pólen } \\
\text { H. polyrhizus } \\
\text { S. setaceus }\end{array}$ & $\begin{array}{l}114,46 \mathrm{a} \\
105,63 \mathrm{a}\end{array}$ & $\begin{array}{l}97,73 \mathrm{a} \\
91,61 \mathrm{a}\end{array}$ & $\begin{array}{l}716,56 \mathrm{a} \\
561,25 \mathrm{~b}\end{array}$ & $\begin{array}{l}250,58 \mathrm{a} \\
218,24 \mathrm{~b}\end{array}$ & $\begin{array}{l}465,98 \mathrm{a} \\
343,01 \mathrm{~b}\end{array}$ & $\begin{array}{l}5,71 \mathrm{a} \\
5,97 \mathrm{a}\end{array}$ & $\begin{array}{l}53,64 \mathrm{a} \\
49,59 \mathrm{a}\end{array}$ \\
\hline $\begin{array}{l}\text { Cobertura } \\
\text { Cobertura clara } \\
\text { Cobertura escura }\end{array}$ & $\begin{array}{l}108,69 \mathrm{a} \\
111,41 \mathrm{a}\end{array}$ & $\begin{array}{l}94,02 \mathrm{a} \\
95,32 \mathrm{a}\end{array}$ & $\begin{array}{l}630,94 \mathrm{a} \\
646,87 \mathrm{a}\end{array}$ & $\begin{array}{l}236,42 \mathrm{a} \\
232,40 \mathrm{a}\end{array}$ & $\begin{array}{l}394,52 \mathrm{a} \\
414,47 \mathrm{a}\end{array}$ & $\begin{array}{l}5,57 \mathrm{a} \\
6,11 \mathrm{a}\end{array}$ & $\begin{array}{l}51,18 \text { a } \\
52,05 \text { a }\end{array}$ \\
\hline $\begin{array}{l}\text { Época } \\
\text { Março } \\
\text { Abril }\end{array}$ & $\begin{array}{l}111,95 \mathrm{a} \\
108,15 \mathrm{a}\end{array}$ & $\begin{array}{l}95,23 \mathrm{a} \\
94,11 \mathrm{a}\end{array}$ & $\begin{array}{l}679,37 \mathrm{a} \\
598,44 \mathrm{a}\end{array}$ & $\begin{array}{l}220,69 \mathrm{a} \\
248,12 \mathrm{a}\end{array}$ & $\begin{array}{l}458,68 \mathrm{a} \\
350,31 \mathrm{~b}\end{array}$ & $\begin{array}{l}4,32 \mathrm{~b} \\
7,36 \mathrm{a}\end{array}$ & $\begin{array}{l}54,41 \mathrm{a} \\
48,82 \mathrm{~b}\end{array}$ \\
\hline Média Geral & 110,05 & 94,67 & 638,9 & 234,41 & 404,5 & 5,84 & 51,61 \\
\hline $\begin{array}{l}\text { Desvio Padrão } \\
\text { CV }(\%)\end{array}$ & $\begin{array}{l}12,36 \\
11.23 \\
\end{array}$ & $\begin{array}{l}9,36 \\
9.89 \\
\end{array}$ & $\begin{array}{c}180,83 \\
28,3 \\
\end{array}$ & $\begin{array}{l}43,67 \\
18,63 \\
\end{array}$ & $\begin{array}{c}147,13 \\
36,37 \\
\end{array}$ & $\begin{array}{r}1,49 \\
25,49 \\
\end{array}$ & $\begin{array}{r}5,94 \\
11,51 \\
\end{array}$ \\
\hline
\end{tabular}

Médias seguidas por letras minúsculas distintas, na coluna, dentro de cada fator de variação, diferem entre si, pelo teste de Tukey, a $5 \%$ de probabilidade. 
TABELA 2 - pH, teor de sólidos solúveis, teor de vitamina C (mg AA/100mL de suco), acidez titulável (g de ácido málico/100mL suco), índice de maturação (SS/AT), e dias até a colheita para frutos de pitaya, resultantes dos cruzamentos realizados, em dois tipos de cobertura e duas épocas. Jaboticabal-SP, 2010.

\begin{tabular}{|c|c|c|c|c|c|c|}
\hline Fator de variação & $\mathrm{pH}$ & SS & Vit. C & $\begin{array}{c}\text { Acidez } \\
\text { Titulável }\end{array}$ & $\begin{array}{l}\text { Índice de } \\
\text { maturação }\end{array}$ & $\begin{array}{r}\text { Dias até a } \\
\text { colheita }\end{array}$ \\
\hline \multicolumn{7}{|l|}{ Origem do pólen } \\
\hline H. polyrhizus & $3,15 \mathrm{a}$ & $12,51 \mathrm{a}$ & $10,56 \mathrm{a}$ & $0,3506 \mathrm{~b}$ & 35,43 a & 39,69 a \\
\hline S. setaceus & $3,28 \mathrm{a}$ & $11,87 \mathrm{a}$ & $9,52 \mathrm{a}$ & $0,4331 \mathrm{a}$ & 29,67 a & 39,56 a \\
\hline \multicolumn{7}{|l|}{ Cobertura } \\
\hline Cobertura clara & $3,12 \mathrm{a}$ & $12,34 \mathrm{a}$ & $10,78 \mathrm{a}$ & $0,3644 \mathrm{a}$ & $35,67 \mathrm{a}$ & $38,94 \mathrm{~b}$ \\
\hline Cobertura escura & $3,32 \mathrm{a}$ & $12,03 \mathrm{a}$ & $9,31 \mathrm{~b}$ & $0,4194 \mathrm{a}$ & 29,43 a & $40,91 \mathrm{a}$ \\
\hline \multicolumn{7}{|l|}{ Época } \\
\hline Março & $3,14 \mathrm{a}$ & $12,14 \mathrm{a}$ & $7,55 \mathrm{~b}$ & $0,4013 \mathrm{a}$ & $30,02 \mathrm{a}$ & $36,00 \mathrm{~b}$ \\
\hline Abril & $3,29 \mathrm{a}$ & $12,23 \mathrm{a}$ & $12,54 \mathrm{a}$ & $0,3825 \mathrm{a}$ & 35,08 a & $43,25 \mathrm{a}$ \\
\hline Média Geral & 3,22 & 12,19 & 10,04 & 0,3919 & 32,55 & 39,62 \\
\hline Desvio Padrão & 0,78 & 0,79 & 1,58 & 0,1049 & 9,89 & 1,76 \\
\hline
\end{tabular}

Médias seguidas por letras minúsculas distintas, na coluna, dentro de cada fator de variação, diferem entre si, pelo teste de Tukey.

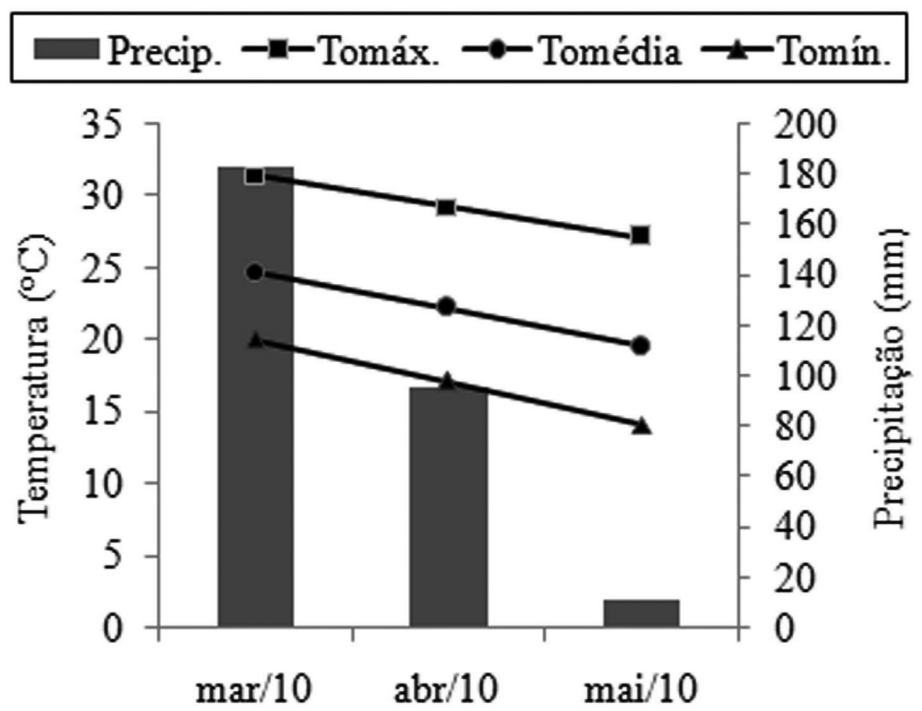

FIGURA 1-Temperaturas máximas, mínimas e médias, e precipitação mensal, durante o desenvolvimento dos frutos de pitaya 'vermelha', no município de Jaboticabal-SP. Dados climatológicos obtidos junto à Estação Meteorológica da FCAV/UNESP, Jaboticabal-SP. 


\section{CONCLUSÕES}

1-Para o clone de H. undatus avaliado, observa-se que não há frutificação, quando autopolinizado.

2-Quando a polinização é feita artificialmente, utilizando-se de pólen de $H$. polyrhizus ou $S$. setaceus, a frutificação é de $100 \%$.

3-O interplantio de $H$. undatus com $H$. polyrhizus ou S. setaceus garante melhor frutificação.

4-A tela plástica de coloração clara influencia positivamente no teor de vitamina $\mathrm{C}$ e no número de dias até a colheita dos frutos.

\section{REFERÊNCIAS}

ANDRADE, R. A.; MARTINS, A. B. G.; SILVA, M. T. H. Influência da fonte de material e do tempo de cura na propagação vegetativa da pitaya-vermelha (Hylocereus undatus Haw). Revista Brasileira de Fruticultura, Jaboticabal, v. 29, n. 1, p. 183-186, 2007.

ANDRADE, R. A.; OLIVEIRA, I. V. M.; SILVA, M. T. H.; MARTINS, A. B. G. Germinação de pitaya em diferentes substratos. Revista Caatinga, Mossoró, v. 21, n. 1, p. 71-75, 2008.

BARBEAU, G. La pitahaya rouge, un nouveau fruit exotique. Fruits, Paris, v. 45, n. 2, p. 141-147, 1990.

CANTO, A. R et al. El cultivo de pitahaya em Yucatan. Yucatán: Universidad Autonoma Chapingo, $1993.53 \mathrm{p}$.

CENTURIÓN, Y. A.; SOLÍS P. S.; SAUCEDO. V. C.; R. BÁEZ. S.; E. SAURI. D.; Câmbios físicos, químicos e sensoriales en frutos de pitahaya (Hylocereus undatus) durante su desarrolo. Revista Fitotecnia Mexicana, Chapingo, v. 31, n. 1, p. 1-5, 2008.

CHITARRA, M. I. F.; CHITARRA, A. B. Pós-colheita de frutas e hortaliças: fisiologia e maneseio. 2. ed. rev. e ampl. Lavras: UFLA, 2005. 785 p.

DONADIO, L. C. Pitaya. Revista Brasileira de Fruticultura, Jaboticabal, v. 31, n. 3, p. 637-929, 2009.
GUNASENA, H. P. M.; PUSHPAKUMARA, D. K. N. G.; KARIYAWASAM, M. Dragon fruit Hylocereus undatus (Haw.) Britton and Rose. In: PUSHPAKUMARA, D. K. N.; GUNASENA, H. P.M.; SINGH, V. P. (Ed.). Underutilized fruit trees in Sri Lanka. Naiobi: World Agroforesty Centre, 2007. p. 110-142.

INSTITUTO ADOLFO LUTZ. Normas analíticas, métodos químicos e físicos para análise de alimentos. 3.ed. São Paulo: IAL, 1985. v.1, 533 p.

LE BELLEC, F. Pollinisation et fecundation de $H y$ locereus undatus et de $H$. costaricensis à I'̂̂le de la Réunion. Fruits, Paris, v. 59, n. 6, p. 411-422, 2004.

LE BELLEC, F.; VAILLANT, F.; INBERT, E. Pitahaya (Hylocereus spp.): a new crop, a market with a future. Fruits, Paris, v. 61, n. 4, p. 237-250, 2006.

LEE, S.K.; KADER, A.A. Preharvest and postharvest factors influencing vitamin $\mathrm{C}$ content of horticultural crops. Postharvest Biology and Technology, Amsterdam, v.20, p.207-220, 2000.

LICHTENZVEIG, J.; ABBO.; S; NERD, A.; TELZUR, N.; MIZRAHI, Y. Cytology and mating system in the climbing cacti Hylocereus and Selenicereus. American Journal of Botany, St. Louis, v. 87, n. 7, p. 1058-1065, 2000.

LONE, A. B.; TAKAHASHI, L. S. A.; FARIA, R. T. Qualidade de frutos de pitaya em função de diferentes fontes de pólen. In: CONGRESSO BRASILEIRO DE FRUTICULTURA, 21., 2010. Natal. Anais...

MARQUES, V. B. Propagação seminífera e vegetativa de pitaia (Hylocereus undatus (Haw.) Britoon \& Rose). 2007. 85 f. Dissertação (Mestre em Agronomia). Universidade Federal de Lavras, Lavras, 2008.

MIZRAHI, Y.; MOUYAL, J.; NERD, A.; SITRIT, Y. Metaxenia in the Vine Cacti Hylocereus polyrhizus and Selenicereus spp. Annals of Botany, London, v. 93, p. 469-472, 2004.

MIZRAHI, Y; NERD, A. Climbing and columnar cacti: new arid lands fruit crops. In: JANICK, J. (Ed.). Perspective in new crops and new crops uses. Alexandria: ASHS, 1999. p. 358-366. 
NERD, A.; SITRIT, Y.; KAUSHIK, R. A.; MIZRAHI, Y. High Summer temperatures inhibit flowering in vine pitaya crops (Hylocereus spp.). Scientia Horticulturae, Amsterdam, v. 96, p. 323-350, 2002a.

NERD, A.; TEL-ZUR, N.; MIZRAHI, Y. Fruits of vine and columnar cacti. In: Cacti, Biology and uses. NOBEL, P. (Ed.). California: University of California Press, 2002b. p. 185-197.

NOMURA, K.; IDE, M.; YONEMOTO, Y. Changes in sugars and acids in pitaya (Hylocereus undatus) fruit development. Journal of Horticultural Science \& Biotechnology, Ashford, v. 80, p. 711-715, 2005.

ORTIZ H, Y. D. Hacia el conocimiento y conservación de la pitahaya (Hylocereus sp.). Oaxaca: Ed. IPN-CONACYT-SIBEJ-FMCN, 2000. 124p.
PUSHPAKUMARA, D. ;DK. N. G.; GUNASENA,H. P. M.; KARYAWASAM, M. Flowering and fruiting phenology, pollination vectors and breeding system of dragon fruit (Hylocereus spp.) Sri Lankan Journal of Agricultural Sciences, Sri Lanka, v. 42, p. $81-91,2005$.

RAVEH, E.; NERD, A.; MIZRAHI, Y. Responses of two hemiepiphytic fruit crop cacti to different degrees of shade. Scientia Horticulturae, Amsterdam, v.73, p. 151-164, 1998.

SILVA, M. A. B. Ceagesp. Disponível em: <mbarros@ceagesp.gov.br>. Acesso em: 03 jan. 2011.

WEISS, J.; NERD, A.; MIZRAHI, Y. Flowering behavior and pollination requirements in climbing cacti with fruit crop potential. HortScience, Alexandria, v. 29 , p. $1.487-1.492,1994$. 